\title{
D-extending modules
}

\author{
Yilmaz Durğun (iD \\ Department of Mathematics, Cukurova University, Adana, Turkey
}

\begin{abstract}
A submodule $N$ of a module $M$ is called d-closed if $M / N$ has a zero socle. D-closed submodules are similar concept to s-closed submodules, which are defined through nonsingular modules by Goodearl. In this article we deal with modules with the property that all d-closed submodules are direct summands (respectively, closed, pure). The structure of a ring over which d-closed submodules of every module are direct summand (respectively, closed, pure) is studied.
\end{abstract}

Mathematics Subject Classification (2010). 16D10, 16D40

Keywords. D-extending modules, d-closed submodules, semiartinian modules

\section{Introduction}

Throughout we shall assume that all rings are associative with identity and all modules are unitary right modules. Let $R$ be a ring and let $M$ be an $R$-module. A (proper) submodule $N$ of $M$ will be denoted by $(N \lesseqgtr M) N \leq M$. By $E(M), \operatorname{Rad}(M)$ and $\operatorname{Soc}(M)$ we shall denote the injective hull, the Jacobson radical, and the socle of $M$ as usual. For undefined notions used in the text, we refer the reader to $[2,11]$.

A submodule $N$ of a module $M$ is essential (or large) in $M$, denoted $N \unlhd M$, if for every $0 \neq K \leq M$, we have $N \cap K \neq 0$; and $N$ is said to be closed in $M$ if $N$ has no proper essential extension in $M$. We also say in this case that $N$ is a closed submodule. Recall that a module is said to be extending or CS or said to satisfy the $C_{1}$ condition if every submodule is essential in a direct summand. Equivalently, every closed (complement) submodule is a direct summand (see, [6]). Extending modules and closed submodules play an important role in rings and modules, and relative homological algebra. See, for example, [6] for their properties. Several generalizations of extending notion and closed submodules have been studied extensively by many authors (see, for example, $[1,4,7-9,14]$ ).

In [10], Goodearl, through Goldie's torsion-free class, defined an s-closed submodule of a module $M$ is a submodule $N$ for which $M / N$ is nonsingular. As a proper generalization of extending modules, Tercan introduced the concept of CLS-modules. Following [20], a module $M$ is called a CLS-module if every s-closed submodule of $M$ is a direct summand of $M$. CLS-modules are recently studied in $[4,14,21]$.

The purpose of the present paper is to introduce and study D-extending modules. Inspired by [10], a submodule $N$ of a module $M$ is called d-closed submodule of $M$ if $M / N$ is in Dickson's torsion-free class i.e. $\operatorname{Soc}(M / N)=0$. We define a D-extending module by the property that every d-closed submodule is a direct summand. We study

Email address: ydurgun@cu.edu.tr

Received: 15.09.2018; Accepted: 07.05.2019 
these modules, generalizing several results both on CLS-modules and CS-modules. It is shown that a ring $R$ is a right C-ring if and only if every injective module is D-extending if and only if every d-closed submodule (of any module) is closed submodule; an $R$-module $M$ is D-extending if and only if $M$ is a C-module and there exists a submodule $M^{\prime}$ of $M$ such that $M=\tau_{D}(M) \oplus M^{\prime}$ and $M^{\prime}$ is a CS module, where $\tau_{D}(M)$ is the largest semiartinian submodule of $M$. Moreover, we proved that every module $M$ with $\operatorname{Soc}(M)=0$ is projective if and only if every module is D-extending. We define, as a generalization of $\Sigma$-CS ring, $\Sigma$-D-extending ring by the property that every module is D-extending. We show that a commutative ring $R$ is a $\Sigma$-D-extending ring if and only if $R$ is a semiartinian ring. Finally, we show that, over a commutative Noetherian ring $R$, every D-closed submodule is pure if and only if $R \cong A \times B$, wherein $A$ is artinian ring and $B$ is hereditary.

\section{Goldie's and Dickson's torsion theories}

Dickson carried the notion of torsion in abelian groups to abelian categories, and he defined in [5] a torsion theory $\tau$ on Mod- $R$ to be a pair $\tau=(\mathcal{T}, \mathcal{F})$ of classes of $R$-modules is called torsion theory if the following three conditions hold:(1) $\operatorname{Hom}(T, F)=0$ for any $T \in \mathcal{T}$ and $F \in \mathcal{F},(2) \mathcal{T}=\{M \mid \operatorname{Hom}(M, F)=0$ for any $F \in \mathcal{F}\},(3) \mathcal{F}=\{M \mid \operatorname{Hom}(T, M)=$ 0 for any $T \in \mathcal{T}\}$. $\mathcal{T}$ and $\mathcal{F}$ called the classes of $\tau$-torsion and $\tau$-torsionfree $R$-modules respectively. The class $\mathcal{T}$ is closed under quotient modules, direct sums and extensions, the class $\mathcal{F}$ is closed under submodules, direct products and extensions. A torsion theory $\tau$ is called the torsion theory generated (cogenerated, respectively) by the class of $R$ modules $\mathcal{M}$ when an $R$-module $F$ is $\tau$-torsionfree ( $\tau$-torsion, respectively) module if and only if $\operatorname{Hom}(M, F)=0(\operatorname{Hom}(F, M)=0$, respectively) for any $M \in \mathcal{M}$. The torsion theory is called hereditary if $\mathcal{T}$ is also closed under submodules. Since $\mathcal{T}$ is closed under homomorphic images and direct sums, there exists a largest submodule $\tau(M)$ of a module $M$ that belongs to $\mathcal{T}$, namely the sum of all submodules of $M$ belonging to $\mathcal{T}$. For each module $M, M / \tau(M)$ is in $\mathcal{F}$.

The singular submodule of a module $M$ is $Z(M)=\{x \in M \mid x I=$ 0 for some essential right ideal $I$ of $R$ \}; this takes the place of the torsion submodule in general setting. The module $M$ is called nonsingular if $Z(M)=0$, and singular if $M=Z(M)$, while the right singular ideal of $R$ is $Z_{r}(R)=Z\left(R_{R}\right)$. The ring $R$ is said to be right nonsingular if it is nonsingular as a right $R$-module. The second singular (or Goldie torsion) submodule $Z_{2}(M)$ of $M$ is defined by the equality $Z_{2}(M) / Z(M)=Z(M / Z(M))$. A module $M$ is called Goldie torsion if $Z_{2}(M)=M$. Let $\tau_{G}$ be the torsion theory generated by the class of singular modules. Then $\tau_{G}$ is a hereditary torsion theory, called the Goldie's torsion theory. Its torsion and torsionfree classes are respectively the class of Goldie torsion modules and the class of nonsingular modules.

A module $A$ is called semiartinian if every non-zero homomorphic image of $A$ contains a simple submodule. Let $\tau_{D}$ be the torsion theory generated by the class of semisimple (or even simple) modules. Then $\tau_{D}$ is a hereditary torsion theory, called the Dickson's torsion theory. Its torsion and torsionfree classes are respectively the class of semiartinian modules and the class of modules which has zero socle. For further information about torsion theories we refer the interested readers to $[2,11]$

\section{D-extending modules}

We begin with the definitions of the main concepts of the paper.

Definition 3.1. A submodule $N$ of a module $M$ is called d-closed (in $M$ ) if $\operatorname{Soc}(M / N)=$ 0 . A module $M$ is called D-extending if every d-closed submodule of $M$ is a direct summand. 
Remark 3.2. Let $M$ be a module.

(1) Let $\tau_{D}(M)$ be a largest semiartinian submodule of $M$. Then, $\operatorname{Soc}\left(M / \tau_{D}(M)\right)=0$, and hence $\tau_{D}(M)$ is a d-closed submodule of $M$.

(2) Every semiartinian module $M$ is a D-extending module. Let $K$ be a d-closed submodule of $M$. Then, $\operatorname{Soc}(M / K)=0$. But the class of semiartinian modules is closed under homomorphic images, and hence $M / K$ is semiartinian. Then $M / K=$ 0 , and this implies $M$ is a D-extending module.

In general, closed submodules need not be d-closed. For example, 0 is a closed submodule of any module $M$, but 0 is d-closed in $M$ only if $\operatorname{Soc}(M)=0$. In contrast to s-closed submodules, d-closed submodules are not closed. Furthermore, injective modules are not D-extending while they are both CLS and extending.

Remark 3.3. Let $M$ be an $R$-module. Then the socle series $\left\{S_{\alpha}\right\}$ of $M$ is defined as: $S_{1}=\operatorname{Soc}(M), S_{\alpha} / S_{\alpha-1}=\operatorname{Soc}\left(M / S_{\alpha-1}\right)$, and for a limit ordinal $\alpha, S_{\alpha}=\cup_{\beta<\alpha} S_{\beta}$. Put $S=\cup\left\{S_{\alpha}\right\}$. Then, by construction $M / S$ has zero socle. $M$ is semiartinian if and only if $S=M$ (see, for example, [6]).

Example 3.4. We give an example for a CS-module that fails to be D-extending. Let $R$ be the ring of all linear transformations (written on the left) of an infinite dimensional vector space over a division ring. Then $R$ is prime, regular, right self-injective and $\operatorname{Soc}\left(R_{R}\right) \neq 0$ by [12, Theorem 9.12]. As $R$ is a prime ring, $\operatorname{Soc}\left(R_{R}\right)$ is an essential ideal of $R_{R}$. Let $S$ be as in Remark 3.3, for $M=R$. Then $S \neq R$, by [3, Lemma 1(2)]. Since $R / S$ has zero socle, $S$ is a d-closed submodule of $R_{R}$. On the other hand, $S$ is not a closed submodule of $R$, otherwise $S$ would be a direct summand of $R$ because $R$ is right self injective (i.e. extending). Therefore $R$ is not a D-extending. Also, as $R$ is right self injective $R^{n}$ is injective, and so extending for every $n \geq 1$.

A ring $R$ is called a right C-ring if $\operatorname{Soc}(R / I) \neq 0$ for every proper essential right ideal $I$ of $R$. Equivalently, $R$ is a right C-ring if every singular module is semiartinian. Left perfect rings, right semiartinian rings, two-sided hereditary Noetherian rings are example for right C-rings. (See $[2,10.10]$ )

Theorem 3.5. Let $R$ be a ring. The following are equivalent:

(1) $R$ is a right $C$-ring.

(2) Every d-closed submodule (of any module) is s-closed.

(3) Every d-closed submodule (of any module) is closed.

(4) Every injective module is D-extending.

(5) For every non-injective module $A, E(A) / A$ is semiartinian.

Proof. (1) $\Rightarrow(2)$ It is enough to show that every module $M$ with $\operatorname{Soc}(M)=0$ is nonsingular. Let $M$ be a module with $\operatorname{Soc}(M)=0$. Assume that $Z(M) \neq 0$. Then $M$ has a nonzero singular submodule and, by (1), $M$ has a simple submodule. But this contradict with $\operatorname{Soc}(M)=0$. Therefore, $M$ is a nonsingular module. (2) $\Rightarrow$ (3) It follows by by $[19$, Lemma 2.3]. (3) $\Rightarrow$ (4) follows by (3) and by fact that injective modules are extending. $(4) \Rightarrow(5)$ Let $A$ be a non-injective module. Assume that $E(A) / A$ is not semiartinian. Then, by definition of semiartinian modules, there is a non-zero module $X$ with $\operatorname{Soc}(X)=0$ and a non-zero epimorphism $f: E(A) / A \rightarrow X$. There is a submodule $U$ of $E(A)$ such that $A \leq U$ and $\operatorname{Ker}(f) \cong U / A$. Note that, by essentiality of $A$ in $E(A), U$ is essential in $E(A)$. Since $\operatorname{Soc}(E(A) / U) \cong \operatorname{Soc}(X)=0$, by $(4), U$ is a direct summand of $E(A)$, a contradiction. (5) $\Rightarrow(1)$ Let $A$ be a module and $B$ a proper essential submodule of $A$ with $\operatorname{Soc}(A / B)=0$. By essentiality of $B$ in $A, E(A)=E(B)$. Since $A / B \leq E(A) / B$, by $(5), A / B$ is semiartinian. Then $\operatorname{Soc}(A / B) \neq 0$. This implies that $R$ is a right $C$-ring. 
Proposition 3.6. Let $R$ be a ring. Every s-closed submodule (of any module) is d-closed if and only if every simple module is singular.

Proof. $(\Rightarrow)$ Obvious. $(\Leftarrow)$ It is enough to show that every nonsingular module $M$ has zero socle. Let $M$ be a nonsingular module with $\operatorname{Soc}(M) \neq 0$. Then $M$ has a simple submodule, say $S$. By nonsingularity of $M, S$ is nonsingular (projective). This contradict with our assumption, and so $\operatorname{Soc}(M)=0$.

A module $M$ is called weakly-flat if if the kernel of any epimorphism $L \rightarrow M$ is closed in the module $L$. Equivalently, every short exact sequence ending with $M$ is closed exact sequence. Weakly-flat modules have been studied in [22]. Every nonsingular module is weakly-flat, and the converse is true if $R$ is a right nonsingular ring by [19, Lemma 2.3]. The following is immediate consequence of Theorem 3.5.

Corollary 3.7. A ring $R$ is a right C-ring if and only if every module in the Dickson's torsionfree class is weakly-flat.

Proposition 3.8. Let $A$ be a module with $\operatorname{Soc}(A)=0$. Then, every closed submodule of $A$ is d-closed.

Proof. Let $B$ be a closed submodule of $A$. Assume that $\operatorname{Soc}(A / B) \neq 0$. Then there is a submodule $C$ of $A$ such that $B$ is a maximal submodule of $C$, i.e. $C / B$ is simple module. Since every maximal submodule is either a summand or an essential submodule and $B$ is closed submodule of $A, C=B \oplus B^{\prime}$, where $B^{\prime} \cong C / B$. But $S o c(A)=0$, and so $0 \neq \operatorname{Soc}(B) \oplus \operatorname{Soc}\left(B^{\prime}\right)=\operatorname{Soc}(B) \oplus B^{\prime}=\operatorname{Soc}(C) \subset \operatorname{Soc}(A)=0$, a contradiction.

Corollary 3.9. A D-extending module with zero socle is a $C S$.

Proposition 3.10. Any direct summand of a D-extending module is a D-extending module.

Proof. Suppose $M=K \oplus K^{\prime}$ for some submodules $K$ and $K^{\prime}$ of $M$. Let $L$ be a d-closed submodule of $K$. Since $\frac{M}{L \oplus K^{\prime}}=\frac{K \oplus K^{\prime}}{L \oplus K^{\prime}} \cong \frac{K}{L}$, then $L \oplus K^{\prime}$ is d-closed submodule of $M$. Then $L \oplus K^{\prime}$ is a direct summand of $M$ which implies that $L$ is a direct summand of $K$. It follows that $K$ is a D-extending module.

Inspired from Theorem 3.5, we will call a right module $M$ is a C-module if every dclosed submodule of $M$ is closed. It is easy to see that every right module is a C-module if and only if $R$ is a right C-ring.

Proposition 3.11. C-modules are closed under quotients.

Proof. Let $M$ be a C-module and $N$ a submodule of $M$. We will show that $M / N$ is a Cmodule. Assume contrarily that there is a d-closed submodule $K / N$ in $M / N$ which is not closed in $M / N$, where $N \leq K \leq M$. Then $\operatorname{Soc}((M / N) /(K / N)) \cong \operatorname{Soc}(M / K)=0$ and there is a submodule $L / N$ in $M / N$ such that $K / N$ is essential in $L / N$, where $K \leq L \leq M$. Since $M$ is a C-module and $\operatorname{Soc}(M / K)=0, K$ is closed in $M$. But, by essentiality of $K / N$ in $L / N, K$ is essential in $L$, a contradiction.

Theorem 3.12. A module $M$ is D-extending if and only if $M$ is a $C$-module and there exists a submodule $M^{\prime}$ of $M$ such that $M=\tau_{D}(M) \oplus M^{\prime}$ and $M^{\prime}$ is a CS module. In this case $M^{\prime}$ is $\tau_{D}(M)$-injective.

Proof. Assume that a module $M$ is a D-extending. $M$ is a C-module by the fact that every direct summand is closed. By our assumption, $\tau_{D}(M)$ is a direct summand of $M$ so that $M=\tau_{D}(M) \oplus M^{\prime}$ for some submodule $M^{\prime}$ of $M$. Note that $S o c\left(M^{\prime}\right)=0$. By Proposition 3.10, $M^{\prime}$ is a D-extending and hence a CS-module by Corollary 3.9. Conversely, suppose 
$M=\tau_{D}(M) \oplus M^{\prime}$ for some CS module $M^{\prime}$. Let $K$ be a d-closed submodule of $M$. Then $\tau_{D}(M) \leq K$ and hence $K=\tau_{D}(M) \oplus\left(K \cap M^{\prime}\right)$. Now $M / K \cong M^{\prime} /\left(K \cap M^{\prime}\right)$ so that $K \cap M^{\prime}$ is a d-closed submodule of $M^{\prime}$. By Proposition 3.11, $M^{\prime}$ is a C-module, and hence $M^{\prime}=\left(K \cap M^{\prime}\right) \oplus K^{\prime}$ for some submodule $K^{\prime}$ of $M^{\prime}$. It follows that $M$ is a D-extending module. The second part is obvious.

Corollary 3.13. A ring $R$ is right $D$-extending if and only if $R$ is right $C$-ring and there exists a direct summand $J$ of $R$ such that $R=\tau_{D}(R) \oplus J$ where $J$ is a CS module.

Corollary 3.14. Let $M$ be a module with $\operatorname{Soc}(M)=0$. Then $M$ is a D-extending if and only if $M$ is a $C S$ module and a $C$-module.

Corollary 3.15. Let $R$ be a ring with $\operatorname{Soc}\left(R_{R}\right)=0$. Then $R$ is a right D-extending if and only if $R$ is a right $C S$-ring and a right $C$-ring. 9].

The following result can be easily proved by the following similar proof of $[20$, Theorem

Theorem 3.16. Suppose that a right $R$-module $M$ is a direct sum $M_{1} \oplus M_{2}$ of D-extending modules $M_{1}$ and $M_{2}$ such that $M_{1}$ is $M_{2}$-injective. Then $M$ is a D-extending.

Let $\mathrm{n}$ be a positive integer and let $M_{1}, M_{2}, M_{3}, \ldots, M_{n}$ be right $R$-modules. Then these modules are called relatively injective if $M_{i}$ is $M_{j}$ injective for all $1 \leq i \neq j \leq n$, see [13]. We have the similar result of [13, Theorem 5] for the finite direct sums of D-extending modules by Theorem 3.16 and Proposition 3.10.

Theorem 3.17. Let $M$ be a module such that $M=M_{1} \oplus M_{2} \oplus M_{3} \oplus \ldots \oplus M_{n}$ is a finite direct sum of relatively injective modules $M_{i}, 1 \leq i \leq n$. Then $M$ is a D-extending module if and only if $M_{i}$ is a D-extending module for each $1 \leq i \leq n$.

Theorem 3.18. The following are equivalent:

(1) Every projective module is D-extending.

(2) Every module $M$ with $\operatorname{soc}(M)=0$ is projective.

(3) Every module is D-extending.

Proof. (1) $\Rightarrow(2)$ Let $M$ be a module with $\operatorname{soc}(M)=0$. Consider the short exact sequence $0 \rightarrow K \rightarrow F \rightarrow M \rightarrow 0$ where $F$ is a projective module. $K$ is d-closed submodule of the projective module $F$ and hence, by (1), the sequence splits. Therefore, $M$ is isomorphic to a direct summand of $F$, and so it is a projective module. (2) $\Rightarrow(3)$ Let $M$ be a module and $N$ a d-closed submodule of $M$. Then $N$ is a direct summand of $M$ since $M / N$ is a projective module. (3) $\Rightarrow$ (1) Obvious.

Oshiro [16] called a ring $R$ a right co-H ring (in honor of Harada) if every projective module is extending. co- $\mathrm{H}$ rings are also called as $\Sigma$-CS rings (see [6]). A right $\Sigma$-CS ring is a left artinian by [17, Proposition 3.2]. Motivated from $\Sigma$-CS ring, a ring $R$ is called a right $\Sigma$-D-extending if the one of the equivalent conditions of Theorem 3.18 are satisfied.

Theorem 3.19. Let $R$ be a commutative ring. The following statements are equivalent:

(1) $R$ is $\Sigma$-D-extending ring.

(2) $R$ is semiartinian ring.

Proof. $(1) \Rightarrow(2)$ By $(1), \tau_{D}(R)$ is direct summand of $R$, i.e. $R \cong A \times B$, where $B=\tau_{D}(R)$ is semiartinian, and $\operatorname{Soc}(A)=0$ as $\operatorname{Soc}(R) \subseteq \tau_{D}(R)$. Without of loss generality, we can assume $R$ is semiartinian or $\operatorname{Soc}(R)=0$. In the later case, $R$ is a $\Sigma$-CS ring by Proposition 3.8 and Theorem 3.18. But $\Sigma$-CS ring is perfect ring, and so $\operatorname{Soc}(R) \neq$. So this case is not possible. $(2) \Rightarrow(1)$ This follows by the fact that every module over a semiartinian ring is semiartinian. 
Proposition 3.20. Let $R$ be a ring. The following are equivalent:

(1) Every d-closed submodule is pure.

(2) Every module $M$ with $\operatorname{Soc}(M)=0$ is flat.

(3) Every finitely generated module $M$ with $\operatorname{Soc}(M)=0$ is flat.

Proof. (1) $\Rightarrow(2)$ Let $M$ be a module with $\operatorname{Soc}(M)=0$. Consider the short exact sequence $0 \rightarrow K \rightarrow F \rightarrow M \rightarrow 0$ where $F$ is a projective module. $K$ is d-closed submodule of the projective module $F$ and hence, by our assumption, the sequence is pure exact. Then, $M$ is a flat module by [15, Corollary 4.86]. (2) $\Rightarrow(3)$ Obvious. (3) $\Rightarrow(1)$ Let $M$ be a module and $N$ a d-closed submodule of $M$. Then $\operatorname{Soc}(M / N)=0$ and, by our assumption, every finitely generated submodule of $M / N$ is a flat module. By [18, Proposition 3.48], $M / N$ is a flat module, and hence $N$ is a pure submodule of $M$ by [15, Corollary 4.86].

Remark 3.21. Let $R$ be a ring and $e$ be a central idempotent in $R$. Then for a right $R$-module $M$ one has, $M=M e \oplus M(1-e)$. It can be easily verified that, $M$ is a flat $R$-module if and only if $M e$ is a flat $e R$-module and $M(1-e)$ is flat $(1-e) R$-module.

Theorem 3.22. Let $R$ be a commutative Noetherian ring. The following statements are equivalent:

(1) Every d-closed submodule is pure.

(2) $R \cong A \times B$, wherein $A$ is artinian ring and $B$ is hereditary.

Proof. (1) $\Rightarrow(2)$ Assume that every d-closed submodule is pure. Then $R / \tau_{D}(R)$ is a flat module, and by Noethernity of $R$, it is a projective module. Therefore $\tau_{D}(R)$ is direct summand of $R$, i.e. $R \cong A \times B$, where $A=\tau_{D}(R)$ is artinian, and $\operatorname{Soc}(B)=0$ as $\operatorname{Soc}(R) \subseteq \tau_{D}(R)$. Without of loss generality, we can assume $R$ is artinian or $\operatorname{Soc}(R)=0$. In the later case, let $I$ be an ideal of $R$. Since $\operatorname{Soc}(R)=0$, we have $\operatorname{Soc}(I)=0$. Then, $I$ is flat by Proposition 3.20. But $R$ is Noetherian, and so $I$ is finitely generated. Therefore $I$ is projective, and so $R$ is hereditary.

$(2) \Rightarrow(1)$ Assume that $R \cong A \times B$, wherein $A$ is artinian and $B$ is hereditary. Let $M$ be a module with zero socle. It is enough to show that $M$ is a flat module by Proposition 3.20. $M=M A \oplus M B, M A$ is an $A$-module and $M B$ is a $B$-module. Since $\operatorname{Soc}(M)=0$, $\operatorname{Soc}(M A)=0$ and $\operatorname{Soc}(M B)=0$. By Theorem 3.18, $M A$ is a projective $A$-module. But since $A$ is artinian, $\operatorname{Soc}(M A)=0$ if and only if $M A=0$. Now consider the short exact sequence $0 \rightarrow K \rightarrow P \rightarrow M B \rightarrow 0$, where $P$ is a projective $B$-module. Since hereditary noetherian rins are C-rings, $K$ is a closed submodule of $P$ by Theorem 3.5. Then $M B$ is nonsingular $B$-module by [19, Lemma 2.3]. By [10, Proposition 2.3], $M B$ is a flat $B$-module. Now, the claim follows by Proposition 3.20.

Acknowledgment. This work was supported by Research Fund of the Çukurova University (Project Number:10871). The author would like to thank the referee for the helpful comments and suggestions.

\section{References}

[1] E. Büyükaşık and Y. Durğun, Neat-flat Modules, Comm. Algebra, 44 (1), 416-428, 2016.

[2] J. Clark, C. Lomp, N. Vanaja and R. Wisbauer, Lifting modules, Birkhäuser Verlag, Basel, 2006.

[3] J. Clark and P.F. Smith, On semi-Artinian modules and injectivity conditions, Proc. Edinburgh Math. Soc. 39 (2), 263-270, 1996.

[4] S. Crivei and S. Şahinkaya, Modules whose closed submodules with essential socle are direct summands, Taiwanese J. Math. 18 (4), 989-1002, 2014.

[5] S.E. Dickson, A torsion theory for Abelian categories, Trans. Amer. Math. Soc. 121, 223-235, 1966. 
[6] N.V. Dung, D.V. Huynh, P.F. Smith and R. Wisbauer, Extending modules. Pitman Research Notes in Math. Ser. 313, Longman Scientific \& Technical, Harlow, 1994.

[7] Y. Durğun, On some generalizations of closed submodules, Bull. Korean Math. Soc. 52 (5), 1549-1557, 2015.

[8] Y. Durğun and S. Özdemir, On S-closed submodules, J. Korean Math. Soc. 54 (4), 1281-1299, 2017.

[9] L. Fuchs, Neat submodules over integral domains, Period. Math. Hungar. 64 (2), 131-143, 2012.

[10] K.R. Goodearl, Singular torsion and the splitting properties. Amer. Math. Soc. 124, Providence, R. I., 1972.

[11] K.R. Goodearl, Ring theory, Marcel Dekker, Inc., New York-Basel, 1976.

[12] K.R. Goodearl, von Neumann regular rings, Monographs and Studies in Mathematics, 4, Pitman Boston, Mass, 1979.

[13] A. Harmanci and P.F. Smith, Finite direct sums of CS-modules, Houston J. Math. 19 (4), 523-532, 1993.

[14] Y. Kara and A. Tercan, When some complement of a z-closed submodule is a summand, Comm. Algebra, 46 (7), 3071-3078, 2018.

[15] T.Y. Lam, Lectures on modules and rings, Graduate Texts in Mathematics, SpringerVerlag, New York, 1999.

[16] K. Oshiro, Lifting modules, extending modules and their applications to QF-rings, Hokkaido Math. J. 13 (3), 310-338, 1984.

[17] K. Oshiro, On Harada rings. I, II, Math. J. Okayama Univ. 31, 161-178, 179-188, 1989.

[18] J.J. Rotman, An introduction to homological algebra., Second, Universitext, Springer, New York, 2009.

[19] F.L. Sandomierski, Nonsingular rings, Proc. Amer. Math. Soc. 19, 225-230, 1968.

[20] A. Tercan, On CLS-modules Rocky Mountain J. Math. 25 (4), 1557-1564, 1995.

[21] J. Wang and D. Wu, When an S-closed submodule is a direct summand, Bull. Korean Math. Soc. 51 (3), 613-619, 2014.

[22] H. Zöschinger, Schwach-Flache Moduln, Comm. Algebra, 41 (12), 4393-4407, 2013. 